\title{
Géoéconomie des rapports firmes-territoires dans le secteur informatique indien
}

Geoeconomical dynamic and reversal of firms-territoiries relationships in Indian ICT sector

Geoökonomische Dynamik und Umkehr der Firma/Gebieten Beziehungen : Der Fall von Informatiktechnologien in Indien

\section{Divya Leducq}

\section{(2) OpenEdition}

\section{Journals}

Édition électronique

URL : http://journals.openedition.org/rge/2860

DOI : $10.4000 /$ rge.2860

ISSN : 2108-6478

\section{Éditeur}

Association des géographes de l'Est

Édition imprimée

Date de publication : 31 octobre 2010

ISSN : 0035-3213

\section{Référence électronique}

Divya Leducq, "Géoéconomie des rapports firmes-territoires dans le secteur informatique indien », Revue Géographique de l'Est [En ligne], vol. 50 / 1-2 | 2010, mis en ligne le 30 septembre 2011, consulté le 08 septembre 2020. URL : http://journals.openedition.org/rge/2860 ; DOI : https://doi.org/10.4000/ rge.2860

Ce document a été généré automatiquement le 8 septembre 2020

Tous droits réservés 


\title{
Géoéconomie des rapports firmes- territoires dans le secteur informatique indien
}

Geoeconomical dynamic and reversal of firms-territoiries relationships in Indian ICT sector

Geoökonomische Dynamik und Umkehr der Firma/Gebieten Beziehungen : Der

Fall von Informatiktechnologien in Indien

\author{
Divya Leducq
}

1 Depuis l'an 2000, à travers les entreprises dont elle est l'hôte, l'Inde s'est affirmée comme le géant mondial des services informatiques et solutions logicielles pour des clients internationaux qui externalisent un certain nombre des tâches nécessaires à leur fonctionnement (Saith et Vijayabaskar, 2005). Une tendance qui continue de s'accentuer comme en témoigne le nombre grandissant d'occurrences trouvées sur la toile par le moteur de recherche Google pour la combinaison sémantique Information Technologies India ${ }^{1}$. Cette intégration dans le système productif mondialisé de l'informatique n'est pour autant pas le fruit de la contingence mais bien le résultat d'une combinaison d'actions que certains nomment géoéconomiques (Lorot, 1999). Ces actions rappellent que la frontière est mince entre l'agent et l'acteur, entre l'État et les entreprises transnationales - qu'elles soient endogènes ou exogènes.

2 La filière indienne des TIC offre du point de vue géoéconomique un terrain d'étude particulièrement intéressant. Le climat d'affaire y a été grandement favorisé par les acteurs publics multiscalaires (Coris et Rallet, 2008). Toutefois, l'inversion des jeux de pouvoir est de plus en plus significative de la part grandissante et souvent indirecte que prennent les entreprises informatiques dans l'aménagement des territoires indiens. De manière inductive, on émet ainsi une hypothèse concernant la dynamique contemporaine du capitalisme. L'évolution des lieux et des relations de pouvoir traduirait le passage d'un État (indien) façonnant l'émergence d'un tissu productif solide $^{2}$ à l'affirmation du positionnement international de ces mêmes entreprises qui 
contribueraient par réflexivité à influencer les multiples échelles de la scène politique. Si les relations entre les acteurs (État central, États régionaux, pouvoirs urbains et métropolitains, firmes, forums) et les territoires évoluent dans le temps, les rôles ne s'annulent pas les uns les autres mais semblent au contraire contribuer parallèlement à la complexification de la gouvernance territoriale (Gumuchian et Pecqueur, 2007).

Néanmoins, pour des raisons de compréhension de l'analyse géoéconomique, nous prenons le parti de distinguer trois étapes qui composeront les trois parties de l'article. Le premier temps est celui de la coévolution des environnements politique, économique et scientifique, pendant lequel l'Etat a fabriqué les territoires de l'innovation informatique en libérant progressivement l'initiative privée. Vient ensuite une période de structuration et de complexification de la scène des acteurs et de leur rôle dans la géoéconomie de l'informatique. Les fleurons sont confortés sur la scène internationale et à travers eux, ce sont les territoires aux échelles intermédiaires régionales et urbaines qui engrangent les profits. Dans le même temps, les entreprises indiennes, devenues firmes multinationales ou plus simplement exportatrices nettes de services informatiques, s'instituent au sein d'une puissante organisation de lobbying, dont le rôle politique va croissant. Le troisième temps est celui de l'inversion des jeux d'acteurs, de dominants et de dominés, de décideurs et d'aménageurs, d'assistants et d'assistés. Les entreprises constituées au sein de plusieurs types de forums revendiquent un certain nombre de mesures d'assouplissement du cadre législatif à leur égard, ce qui entraîne des conséquences plus ou moins favorables pour le développement structurel des territoires.

4 Cet article utilise des éléments réunis lors de séjours de recherche effectués en Inde entre mars 2008 et juin 2009. Nous avons exploité à la fois des données macrogéographiques à l'échelle de l'Union indienne et des données micro-géographiques à l'échelle des États et des aires urbaines. Les informations ont été collectées directement auprès des agences et acteurs publics et privés de la filière des services informatiques en Inde. Les données quantitatives recueillies auprès du Ministère des Technologies de l'Information et de la Communication, du Ministère du Commerce et de l'Industrie, ainsi que NASSCOM, le syndicat patronal des entreprises de services informatiques indiennes ${ }^{3}$ nous ont permis de produire un certain nombre de cartes chronologiques ou thématiques. Ces dernières expriment des valeurs absolues. Les Plans Quinquennaux indiens sont disponibles en ligne pour des raisons recherchées de transparence et d'accessibilité de la démocratie pour tous les citoyens ${ }^{4}$. Le reste des analyses a été effectué grâce à l'exploitation de récits de vie et d'entretiens semi-directifs menés auprès de grandes entreprises et de start-ups enquêtées dans les métropoles régionales de Pune (Maharashtra), Thiruvananthapuram et Kochi (Kerala). Des échanges ont aussi eu lieu avec des groupes cibles lors des réunions des réseaux sociaux intervenant dans la gouvernance territoriale des activités numériques. Nous avons pu entrer en contact avec les acteurs des TIC indiennes en valorisant la bonne connaissance de leur organisation et la méthode de proche en proche ${ }^{5}$. 


\section{Coévolution des environnements politique et scientifique : un État central maître d'ouvrage}

\section{A. Cohérence et évolution des plans quinquennaux}

Nombreux sont les auteurs qui ont démontré que la puissance publique joue un rôle essentiel dans le cadre de la remontée de filière d'une nation (Glaeser, 2005 ; Tatemi, 2005 ; Depret et Hamdouch, 2007), par sa capacité à mettre en place des cadres politiques, législatifs, scientifiques et technologiques suffisamment solides pour permettre à la fois la naissance et l'ancrage d'une filière d'activité dans un pays dont les conditions ne le prédisposaient pas à devenir un géant des hautes technologies. Ainsi la lecture de la coévolution des environnements institutionnels, scientifiques et économiques à travers des plans quinquennaux indiens de 1951 à 2012 (fig. 1), permet de comprendre en quoi ces douze documents de prospective économique et territoriale ont et continuent de constituer le socle solide des orientations prises par et pour le secteur des technologies de l'information et de la communication. Dès le Sixième Plan et après de nombreux signes avant-coureurs dans les précédents documents édités par la Commission au Plan ${ }^{6}$, Rajiv Gandhi annonce la New Computer Policy en 1984, suivie de la création en 1985 d'un Department of Telecommunication, et en 1987 d'une nouvelle réglementation favorable aux exportations des produits TIC et à la formation d'une main d'œuvre compétente. Dans le même temps (1986-1996) les réseaux de télécommunications sont développés à travers le pays et leur gestion est rationalisée par la création de deux compagnies, la Mahanagar Telephone Nigam Limited pour les communications nationales et la Videsh Sanchar Nigam Limited (VSNL) qui a détenu le monopole des communications internationales jusqu'en 2002. La VSNL fut chargée, à partir de 1995, de mettre en place l'accès à l'Internet commercial.

Figure 1 : Chronologie des plans quinquennaux indiens

$$
\begin{array}{llllll}
\mathbf{1}^{\text {er }} \text { plan } & 1951-56 & \mathbf{5}^{\mathbf{e}} \text { plan } & 1974-79 & \mathbf{9}^{\mathbf{e}} \text { plan } & 1997-2002 \\
\mathbf{2}^{\text {nd }} \text { plan } & 1956-61 & \mathbf{6}^{\mathbf{e}} \text { plan } & 1980-85 & \mathbf{1 0}^{\mathbf{e}} \text { plan } & 2002-07 \\
\mathbf{3}^{\mathbf{e}} \text { plan } & 1961-66 & \mathbf{7}^{\mathrm{e}} \text { plan } & 1985-90 & \mathbf{1 1}^{\mathrm{e}} \text { plan } & 2007-12 \\
\mathbf{4}^{\mathbf{e}} \text { plan } & 1969-74 & \mathbf{8}^{\mathrm{e}} \text { plan } & 1992-97 & \mathbf{1 2}^{\mathrm{e}} \text { plan } & 2012-17
\end{array}
$$

6 Quatre chapitres politiques des Plans concourent à élaborer un cadre institutionnel favorable aux entreprises informatiques: le chapitre consacré aux technologies de l'information et aux télécommunications, le volet sectoriel industries et services informatiques, la partie sur l'enseignement supérieur et celle dédiée au développement des sciences et technologies. On dresse ici, de manière synthétique, les évolutions principales relevées au sein de ces chapitres entre le Premier et le Onzième Plan Quinquennal indien. De manière récurrente on note que les idées et la rhétorique des Plans portaient au début sur la nécessité d'une mise à niveau des infrastructures et des personnels et que les discours actuels ambitionnent par des mesures novatrices de talonner - voire de dépasser - les territoires informatiques les plus innovants et les plus performants à l'échelle mondiale (produit logiciel, services aux entreprises et aux particuliers, électronique). Concernant les TI, tandis que les premiers plans 
s'intéressaient à la connectivité sans fil du territoire indien (satellites, WLAN), les derniers Plans incitent les concepteurs de logiciels et de services informatiques à se mettre en relation avec les créateurs, afin de ne plus dissocier la technologie des contenus, mais d'en permettre une évolution conjointe. La politique industrielle, mises à part la libéralisation et la privatisation progressive de l'économie, a vu naître une sous-section dédiée complètement à l'économie servicielle. La valorisation des succès nationaux en informatique et des fleurons reflets d'une identité et d'un savoir-faire indiens est aussi un objectif récurrent des Plans. C'est ainsi que l'on constate dans les trois derniers Plans, l'apparition et la valorisation du capital-risque (business angels) indispensable à l'essor des start-up innovantes. Tandis que les Plans concernant la recherche en Sciences et Technologies ne semblent pas avoir privilégié la voie du software à celle du hardware, l'autonomie de la défense, l'éducation pour tous et l'emploi pour le plus grand nombre sont des leitmotiv pour lesquels le secteur informatique constitue un chemin privilégié d'accès. Concernant enfin l'enseignement supérieur, le gouvernement indien s'est tout d'abord attaché à réduire les disparités très fortes entre certaines universités de piètre qualité - tant en pédagogie qu'en recherche - et les instituts d'excellence nationale ${ }^{7}$. Enfin, l'un des objectifs du Plan Quinquennal en cours est l'autonomisation progressive des universités régionales et d'État, par la création de fondations et de chaires d'excellence privées afin d'assurer la compétitivité de la recherche indienne.

\section{B. Les justifications discursives de New Delhi}

7 La position de l'Inde dans les sciences et technologies a changé au cours des siècles. Pensées, capacités et techniques furent pendant le Moyen-Âge indien parmi les plus avancées du monde alors que l'Inde contemporaine s'attache à rattraper son retard en votant depuis l'Indépendance des politiques visant à accroître ses capacités technologiques. R.A. Mashelkar (2008) rappelle que la croyance dans les sciences et les technologies comme moteur de développement s'appuie sur quatre piliers en Inde : le nationalisme scientifique, la mondialisation technologique, le leadership mondial et la croissance inclusive.

8 Le nationalisme technologique est pour partie du à ce que d'éminents scientifiques aient participé à l'accès à l'Indépendance de l'Inde et à la construction de la période postcoloniale. L'Inde notamment à cause du principe de l'hindutva ${ }^{8}$ imposant un contrôle strict sur les importations n'a pas eu accès à la technologie nécessaire pour son développement. De ce fait, dans certains domaines comme la défense, l'énergie atomique ou la météorologie, des programmes spécifiques ont été financé par New Delhi pour combler les manques. C'est ainsi que les superordinateurs se sont développés et que le C-DAC ${ }^{9}$ a construit de 1987 à 1998 à Pune un superordinateur nommé PARAM. La volonté d'inscrire l'Inde dans la mondialisation technologique remonte à la Politique des Sciences et Technologies de 1958. L'Inde montrait ainsi qu'elle souhaitait à la fois trouver sa place dans la globalisation croissante de l'économie de la connaissance et prendre part à l'internationalisation de la production des technologies, en ouvrant ses portes aux centres de recherche et développement et en publiant de plus en plus d'articles scientifiques. La création d'un écosystème favorable à l'innovation va de paire avec la volonté de leadership mondial dans le domaine des hautes technologies. Elle met en place une série de mesures pour améliorer le droit lié au protocole Internet, augmenter la disponibilité en capital d'amorçage, promouvoir 
des Silicon Valley dans un contexte de circulation des cerveaux et de retours d'un grand nombre de scientifiques et d'ingénieurs dans le pays. La création de cinq Indian Institute of Science Education and Research, huit nouveaux IIT et de vingt Indian Institute of Information Technologies va dans le sens d'un renforcement de la dynamique de recherche en informatique. Par ailleurs, le rôle croissant des instituts de recherche entièrement financés par le secteur privé est à la fois le signe d'un intérêt commun entre la puissance publique et les firmes et celui du désengagement latent de l'État. Les firmes Reliance, Birla ou Tata investissent massivement dans la Recherche \& Développement indienne ${ }^{10}$.

9 Le mythe de la croissance inclusive, c'est en quelque sorte la légitimation démocratique d'une politique d'envergure menée en faveur des sciences et technologies, et plus particulièrement de l'informatique. L'agenda politique officiel est d'améliorer le quotidien de millions d'Indiens issus de toutes les couches sociales, grâce aux innovations incrémentales et radicales produites dans le pays. Cette idée nehruvienne tient à ce que l'informatique ne soit pas juste un levier de développement économique mais constitue une opportunité de transformation qualitative de la société. Ainsi, les crores $^{11}$ de roupies indiennes investies par la puissance publique pour le développement des accès Internet ne servent pas uniquement les intérêts d'une partie du secteur tertiaire - représentée par les sociétés de services en ingénierie informatique (SSII) mais aussi les secteurs d'activité traditionnels. La justification vis-à-vis du quotidien de la société civile se trouve dans l'essor exigé par New Delhi des programmes «egovernance ${ }^{12}$ » qui rend l'administration locale plus accessible et «e-education » qui offre un accès gratuit à la connaissance.

\section{Un aménagement du territoire au service de la technologie}

10 L'agence autonome Science Technology Park of India a été crée par décret gouvernemental en 1990. Elle est placée sous l'égide du Ministère de la Communication et des Technologies de l'Information, qui héberge sa direction générale à New Delhi. L'inscription sur les registres du STPI donne droit à de multiples exemptions fiscales allant de l'installation dans une zone estampillée "STPI» au dégrèvement partiel ou total des bénéfices à l'exportation. L'adhésion à ce régime ouvre aussi la voie à l'accélération des démarches administratives et logistiques nécessaires au fonctionnement des sociétés de services informatiques. Cette politique, décidée par l'État central, dépasse la simple mise en œuvre des franchises, et vise à travers l'aménagement du territoire à consolider le développement de la filière informatique et des entreprises de nationalité indienne tournées vers les marchés extérieurs. En effet, le STPI recouvre dans son opérationnalité des formes de zonage diversifiées, allant du simple immeuble de bureaux en centre-ville à la zone d'activité monofonctionnelle en première ou deuxième couronne de banlieue urbaine.

11 En deux décennies, le STPI est devenu l'interlocuteur privilégié des entreprises de services informatiques et de solutions logicielles indiennes, et ce pour au moins trois raisons. Le STPI démontre la volonté de l'État central de ne plus taxer les profits des firmes et de ne plus entraver les embryons d'entreprenariat local, contrairement au Licence Raj ${ }^{13}$ en place depuis l'Indépendance (Jaffrelot, 2006). En plus de ne pas limiter les initiatives privées, cette institution va aussi encourager le tissu endogène des startup, accompagnant ainsi les entreprises et le discours de l'État indien sur la nécessité 
pour des territoires urbains et régionaux de s'insérer - par l'entrée de gamme puis par l'innovation - dans la compétition économique mondialisée. Enfin, le STPI permet par ses implantations progressives en région de mailler le territoire et de jouer le jeu de deux desseins politiques : favoriser le rééquilibrage d'un territoire dominé par quatre mégapoles - Delhi, Mumbai, Kolkata et Chennai - et renforcer parallèlement les points forts du système national de l'informatique. Une analyse approfondie des Rapports Annuels publiés par cette agence entre 1991 et 2009, ainsi que les entretiens menés auprès de différents directeurs des représentations du STPI dans les villes indiennes ${ }^{14}$, permettent de comprendre quelles sont les étapes structurantes de cette politique d'aménagement du territoire (fig. 2) au service du développement des activités informatiques. Les deux cartes suivantes montrent les installations des agences urbaines du STPI et mettent en relief deux décennies de politiques publiques de soutien territorial aux SSII indiennes. 
Figure 2 : Les étapes de diffusion du STPI (1991-2011)

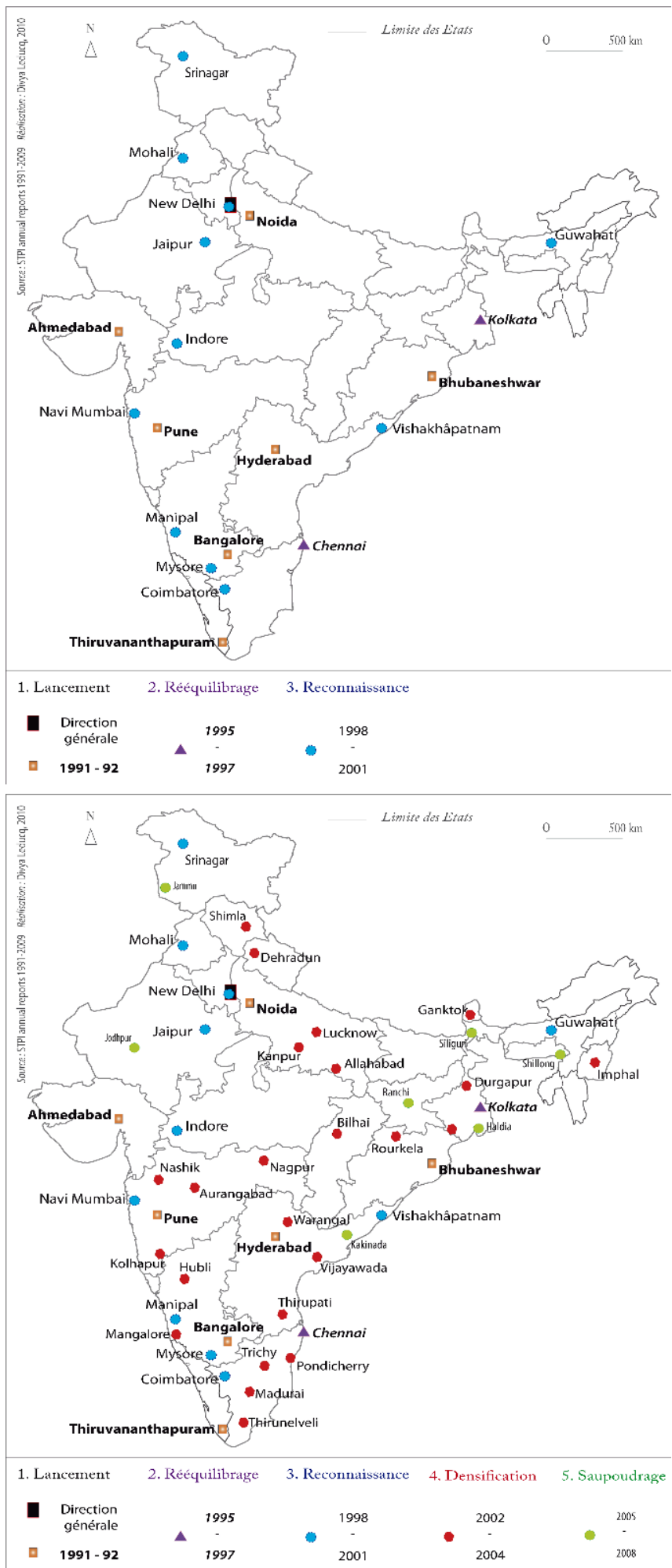

12 La première période est celle du lancement de la politique du STPI, de 1990 à 1992. Elle illustre le fait que le pouvoir central souhaitait favoriser l'émergence de pôles urbains secondaires - dédiés à l'économie numérique - et capable de contrebalancer le poids hérité des anciennes métropoles dont le pouvoir reste lié soit à un tertiaire de commandement soit à une industrialisation précoce. Ainsi, Pune plutôt que Mumbai fût 
choisi dans la Région du Maharashtra. Bangalore a été instauré comme l'une des directions régionales du STPI en raison des nombreuses institutions de haute(s) technologie(s) qui ont germé dans une ville éloignée des frontières pakistanaises et chinoises. La ville nouvelle de Noida, les aires urbaines de Bhubaneshwar, Ahmedabad, Thiruvananthapuram ou encore Hyderabad constituent autant de contrepoids dans la hiérarchie urbaine indienne. La seconde période, même si elle intervient plus tardivement, entre 1995 et 1997, contribue uniquement à rééquilibrer pour des raisons politiques la dynamique TIC insufflée dans les aires secondaires, en établissant une direction du STPI à Chennai (Tamil Nadu) et à Kolkata (Bengale Occidental). La troisième période (1998-2001) voit la reconnaissance des dynamiques territoriales existantes, autour des grandes capitales des TIC comme Bangalore (Manipal, Mysore, Coimbatore), mais aussi la reconnaissance des dynamiques endogènes liées à des spécificités portuaires et logistiques (Vishakhâpatnam), bancaires (Navi Mumbai), et enfin la reconnaissance d'un territoire indien dont l'identité et l'unité sont aussi affirmées à travers la politique du STPI (aux frontières septentrionales notamment à Mohali, Srinagar, Guwahati). Le quatrième temps de la politique (2002-2004) marque une accélération de la densification du maillage national avec l'implantation notamment en Inde du sud de vingt-cinq directions du STPI. La dernière phase de ce programme marque son essoufflement (2005-2008), la fin étant annoncée pour mars 2011. Quelques agences ont été implantées au nord du pays, pour tenter de " masquer » la préférence des entreprises de services informatiques pour le sud et l'ouest.

Cette première partie nous a permis de comprendre le rôle prééminent de la puissance publique dans le rattrapage technologique indien. Depuis l'Indépendance, les TIC sont perçues comme l'un des moteurs du développement économique. La stratégie d'aménagement menée à travers le STPI démontre une volonté constante de rééquilibrage entre des territoires inégaux, or il semble bien que les logiques capitalistes de localisation tendent à la concentration des intérêts privés sur le territoire et à la création - de fait - de pôles de croissance.

\section{Structuration des systèmes territoriaux de l'informatique : la complexification croissante de la scène géoéconomique}

\section{A. Les régions qui gagnent ${ }^{15}$}

14 Si la dynamique du système territorial indien des TIC est ancienne, il s'avère cependant que le poids des régions est inégal (fig. 3). On remarque en effet, en 2008, que si presque tous les États peuvent se targuer de quelques millions d'euros de revenus générés par les SSII présentes sur leur territoire, une nette surreprésentation des Régions du sud et de l'ouest de l'Inde est à noter. Ainsi, l'État de la Silicon Valley de l'Inde, Bangalore, exportait en 2008 plus de 9590 millions d'euros de solutions informatiques et logiciels. Les Régions de la Capitale Nationale, de l'Andhra Pradesh et du Tamil Nadu suivent avec des revenus respectifs aux alentours des 5000 millions d'euros. Loin derrière, se trouve le Bengale Occidental, territoire régional de la capitale économique et administrative de l'époque coloniale ${ }^{16}$. 
Figure 3 : Revenus générés en région par les exportations de services informatiques (2008)

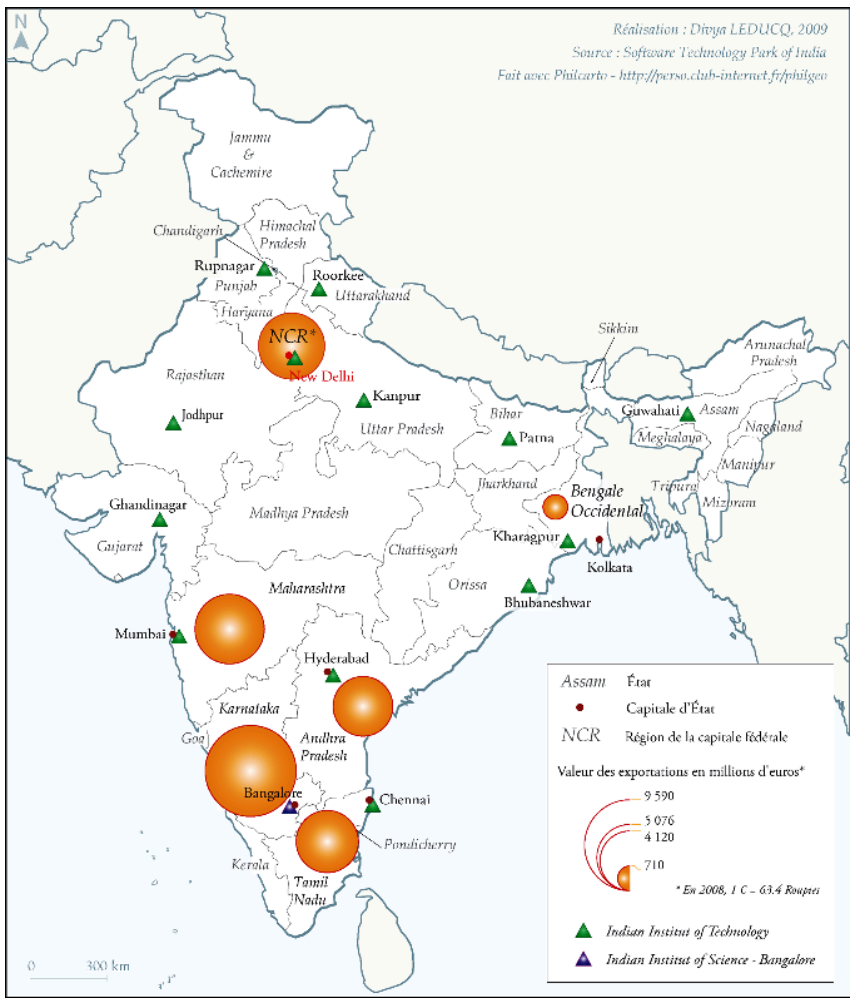

Sur la figure 3, figurent aussi les IIT, Instituts Technologiques d'importance nationale, ainsi que l'Indian Insitute of Science ${ }^{17}$ de Bangalore (IISc). Leur importance est indéniable dans la capacité à générer une élite entrepreneuriale et dans la dynamique de création de firmes multisites, qui prennent très souvent leur enracinement dans la Silicon Valley américaine ou dans les pays du Golfe persique. Les mobilités de ces diplômés qui forment des réseaux très puissants d'alumnis ${ }^{18}$ - sont à la fois internationales mais ont également lieu de manière très importante à l'intérieur de l'Inde. Elles sont fonction du concours d'entrée à l'IIT obtenu mais aussi des opportunités professionnelles qui se présentent. Pour cette première raison de mobilité géographique des ingénieurs diplômés des IIT, on ne peut mettre directement en corrélation la politique de développement de l'excellence scientifique et la performance exportatrice des États. Il existe au moins deux autres raisons qui ne permettent pas d'établir un effet de causalité pour l'ensemble des États. Les IIT n'ont pas tous la même ancienneté. Ainsi les IIT de Kharagpur, Mumbai, Chennai, Kanpur et Delhi ont été fondés dans les années 1950-1960 alors que les autres IIT ont été créés à partir du milieu des années 1990. Enfin, la dernière raison tient dans le fait que les IIT aient développé des spécialisations différentes, ainsi Mumbai et Delhi et l'IIS de Bangalore sont les plus spécialisés dans les technologies informatiques.

La relative dispersion territoriale des revenus informatiques est donc aussi liée au fait que l'ensemble des États régionaux de l'Inde, y compris les moins bien dotés industriellement ou scientifiquement - ont voté des politiques sectorielles soutenant le développement du secteur informatique. Ainsi, par exemple le Kerala, État rural et parallèlement très urbanisé de l'Inde du sud a mis en place dès 1991 et dans la lignée du 8ème Plan Quinquennal des parcs scientifiques dédiés aux activités informatiques. Les politiques régionales se font de plus en plus pragmatiques et évoluent au gré des 
paradigmes technologiques. Ainsi l'État du Maharashtra mettaient en application sa IT and ITES Policy en 2003 et discutait au Parlement régional de l'Animation and Gaming Policy en $2010^{19}$. Parallèlement, la très large acceptation du concept de parc scientifique tend à le vider de son sens. Les États profitent en effet des mesures sectorielles décidées par New Delhi, pour se lancer dans la compétition. Ils créent conjointement avec le STPI des parcs d'activités monofonctionnels en valorisant davantage les liens physiques avec les universités - route reliant le parc à l'université ou zone située sur le campus universitaire - que la proximité cognitive susceptible d'engendrer des collaborations et de l'innovation. Dans les plus grandes villes, on assiste toutefois à la valorisation du potentiel de recherche technologique existant, et au montage de soutiens institutionnels complexes - issus de l'initiative des Chambres de Commerce et d'Industrie - soutenant de ce fait le tissu entrepreneurial endogène. La création d'écoles spécialisées, en partenariat avec des centres étrangers reconnus, allant des États nationaux aux campus universitaires, dans les TIC ou sur les contenus participent aussi à la structuration des milieux régionaux d'innovation informatique.

\section{B. Le poids des aires urbaines, juste révélateur des firmes fabriquant le territoire?}

$\mathrm{Si}$, en période de mondialisation, le rayonnement international d'une entreprise renforce le prestige d'une nation, il semble aussi - et peut-être avant tout - qu'il soit le révélateur du poids des firmes pour les agglomérations urbaines. En effet, les villes, par leur investissement dans le marketing territorial et par l'importance des acteurs privés dans l'économie et la politique locale, paraissent s'identifier à un potentiel d'innovation endogène ou à territoire d'entreprise particulier. Ainsi, au milieu des années 1990, il importait encore assez peu d'avoir pour son bassin d'emploi des firmes comme Infosys, Wipro ou Tata Consultancy Services. A la fin des années 2000, les trois fleurons des services informatiques indiens sont devenus des arguments de marchandisation du territoire incontournables, favorisant indéniablement les phénomènes d'agglomération des entreprises et d'essaimage technologiques. De ce fait, le nombre de sièges sociaux des firmes informatiques est révélateur de l'attractivité des villes (fig. 4). L'agglomération devient le fait de stratégies économiques isolées et ne sont plus la conséquence des mesures politiques incitatives ${ }^{20}$ quand les effets de réputation et de renommée du lieu sont suffisamment importants pour que les localisations d'entreprises soient générées par un effet de mimétisme. Ce besoin de visibilité produit de nouvelles formes de rivalités territoriales, car les acteurs urbains et économiques souhaitent que cette agglomération en cascade finissent par produire des externalités résiliaires leur permettant de devenir le terreau fertile de clusters innovants (Vicente, 2005). 
Figure 4 : Localisation des sièges sociaux des SSII en Inde (2009)

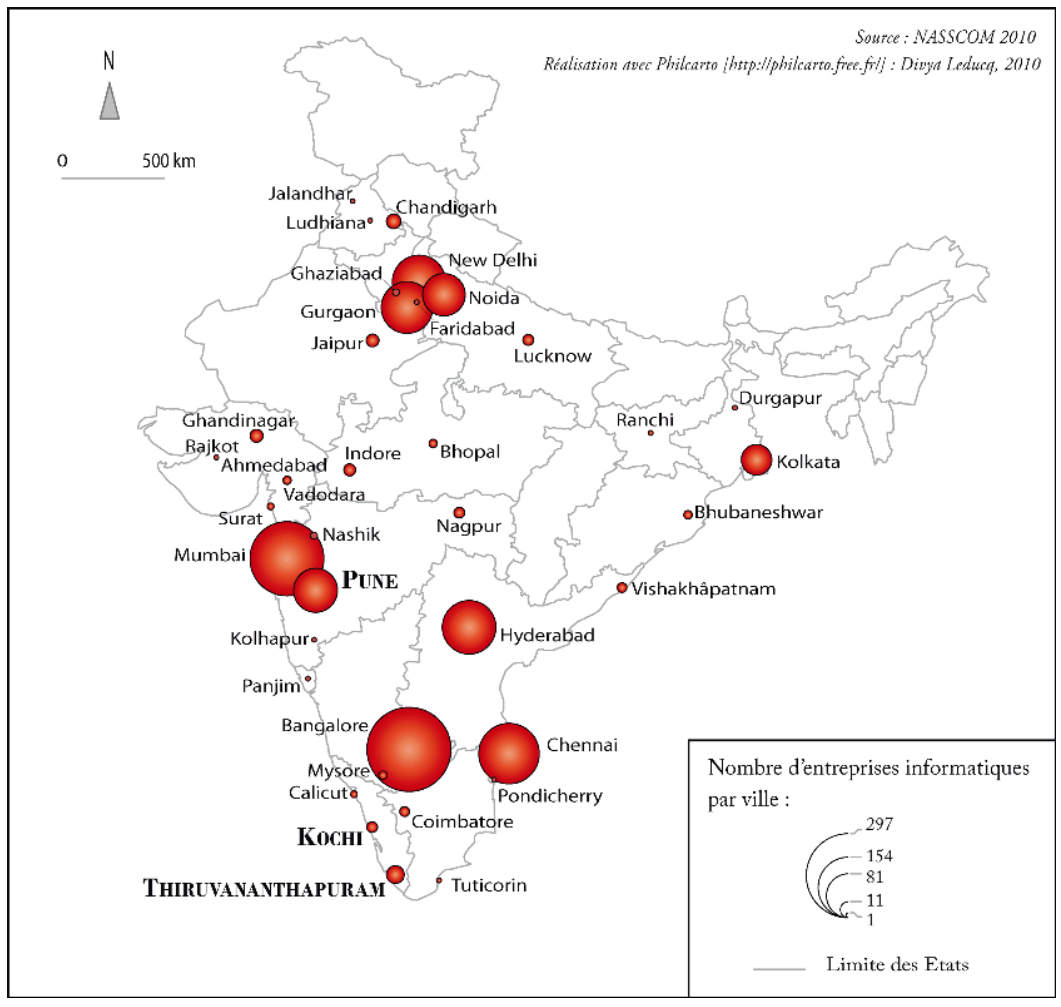

18 La carte des sièges sociaux des SSII révèle sensiblement les mêmes tendances que la carte des revenus régionaux des TIC, si ce n'est qu'elle permet en plus de relativiser le poids des capitales d'État au profit du rôle croissant des villes secondaires de la hiérarchie urbaine indienne. En termes de revenus urbains, ce phénomène est encore plus saillant. Pune dans l'État du Maharastra en est le meilleur exemple. Elle est la septième aire urbaine la plus peuplée de l'Inde et se retrouve, depuis 2009, en quatrième position du chiffre d'affaire des exportations générées par les services informatiques, devançant ainsi Mumbai, la capitale économique et financière de l'Union (Leducq, 2009). Kochi-Ernakulam tend aussi à une échelle économique inférieure à confirmer ce processus de retournement spatial de la hiérarchie urbaine. Le produit de ses exportations de services informatiques dépasse celui de Thiruvananthapuram, capitale politique de l'État du Kerala.

\section{La puissance d'un lobby où la vente des savoirs-faire indiens sur la scène internationale}

La remontée de filière des entreprises indiennes est ainsi symbolisée, organisée et structurée par des lobbys, qui trouvent leur ancrage à la fois sur le sol indien et sur le sol californien de la Silicon Valley. C'est le cas du puissant groupe de pression National Association of Software and Services COMpanies (Nasscom) qui contribue à construire la renommée internationale de l'Inde en produisant un discours accompagné de cartes et de graphiques sur la place de ce pays dans le système productif mondialisé des TIC. NASSCOM balaye ainsi tous les secteurs des services informatiques allant de l'infogérence (opération de Business Process Outsourcing ${ }^{21}$ ) aux innovations logicielles pour des secteurs clés de l'économie comme le nucléaire, l'espace, la bioinformatique, 
la pharmacie, la défense, les technologies vertes, la culture, etc. Apparaît ainsi la collusion entre les intérêts de l'État et ceux du secteur privé dont les agendas apparaissent dès lors compatibles (fig. 5).

Figure 5 : Agenda officiel du lobby Nasscom

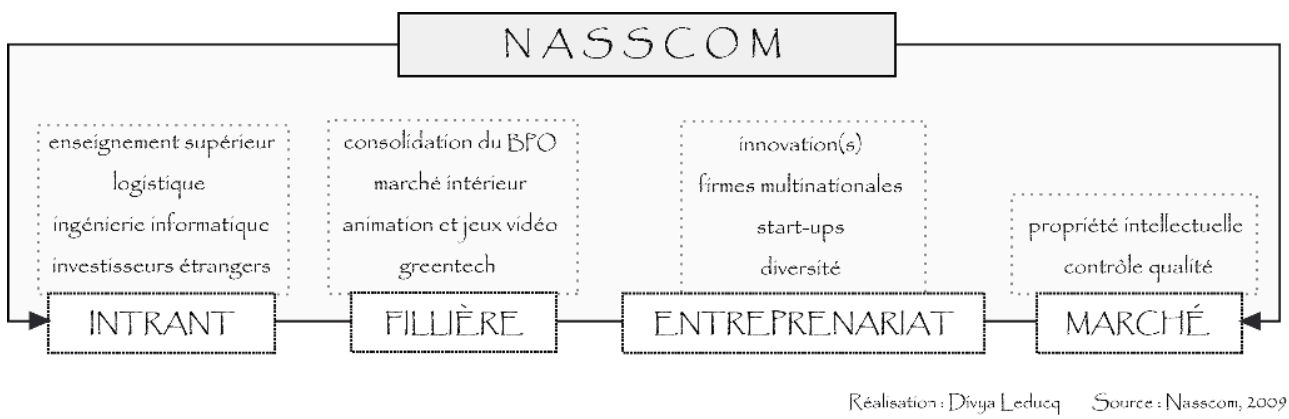

En inscrivant les firmes indiennes dans le paradigme du «glocal » et en influençant les orientations prises en matière de modèle de développement urbain (Nasscom, 2009), ce lobby contribue à l'accentuation de l'insertion grandissante des élites géoéconomiques dans les affaires de l'État et de la cité.

Nous avons pu constater dans cette deuxième partie que le système indien de production des services est à la fois complexe et dynamique. Complexe car les SSII sont dispersées dans toutes les Régions indiennes et concentrées dans le même temps dans les aires métropolitaines de rang A et de plus en plus souvent de rang B. Dynamique, parce que les entreprises à travers de puissants lobbies institués concourent à la promotion territoriale de l'Inde. Ce retournement progressif de la gouvernance des milieux productifs est à la fois favorable à la dynamique entreprenariale - la confiance dépasse les déductions fiscales immédiates - mais pose de nouvelles questions quant à la relation des entreprises aux territoires urbains.

\section{Glissement du pouvoir et inversion progressive des rôles public-privé : quelles implications pour le développement des territoires?}

\section{A. L'ancrage territorial des zones économiques spéciales (ZES)}

Les firmes jouent un rôle multiscalaire important mais inégal dans le fonctionnement et l'aménagement des territoires, et cela est d'autant plus vrai que l'économie urbaine est favorisée par la création de zones franches. En 2005, le Gouvernement indien a mis en place la politique des Zones Economiques Spéciales dans le but d'encourager la prise en charge par le secteur privé de l'aménagement des zones dédiées à la production d'industries et de services (Cadène, 2008). Ainsi, les promoteurs des ZES des TI bénéficient de franchises pour l'acquisition des terrains, la réalisation du gros œuvre (promoteurs), les équipements (co-promoteurs), la construction, l'achat ou la location des bureaux (société de gestion ou SSII). Dès 2003, Nasscom avait encouragé la promulgation d'une telle loi, affirmant les bienfaits de la prise en main par le privé de son destin économico-territorial (Leducq, 2009). A la suite de la parution des décrets nationaux et régionaux d'application de la loi dite des " $S E Z^{22}$ ", Nasscom co-rédige avec des cabinets d'expertise - plusieurs rapports dans lesquels ils recommandent et 
classent des villes et/ou quartiers plus ou moins favorables à un investissement fructueux. Ces actions de lobbying orientent la destination des capitaux et ont deux conséquences spatiales importantes. La première est plutôt positive car elle tend à renforcer le poids des aires urbaines montantes dans la dynamique des TIC. La seconde, plutôt négative, accentue le déséquilibre spatial entre les espaces intégrés et les espaces délaissés de l'économie numérique indienne (fig. 6).

Figure 6 : Couverture territoriale des ZES dédiées aux TIC (2006-2010)

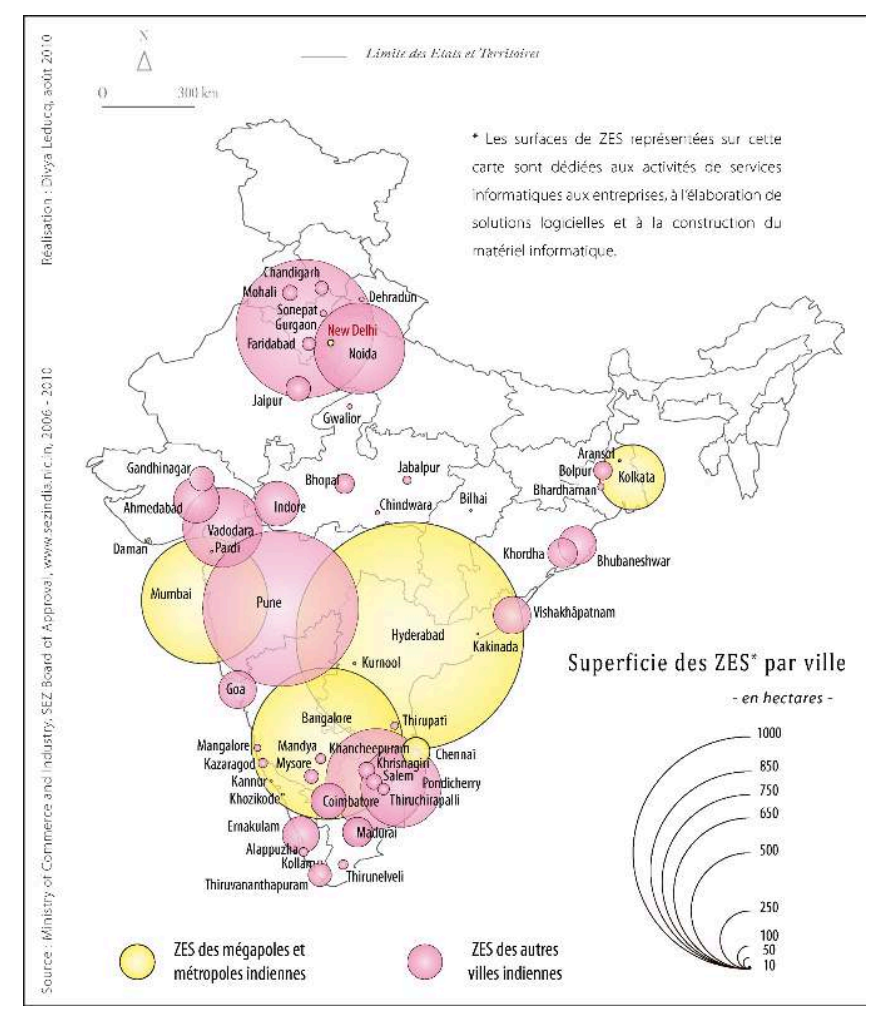

Ainsi, cette cartographie de la superficie par villes des $\mathrm{ZES}^{23}$ cherche à mettre en évidence plusieurs phénomènes territoriaux paradoxaux. Loin d'encourager une couverture homogène du territoire, les largesses fiscales attribuées aux développeurs de ZES encouragent la concentration des capitaux sur des points nodaux du territoire qui connaissent déjà une forte croissance dans le domaine des TIC et qui assurent donc une rentabilité aux promoteurs et aux investisseurs (Kennedy, 2009). Néanmoins, si les franges des mégapoles informatiques - Hyderabad et Bangalore - sortent renforcées, ce n'est pas le cas des mégapoles historiques - Mumbai, Chennai, Kolkata et Delhi. En effet, ce sont les villes centres des métropoles régionales (Pune, Vadodara) ou les villes satellites et périphériques des mégapoles informatiques qui sortent grandes gagnantes de l'émergence de projets de ZES consacrées aux TIC, que ce soit dans l'État du Kerala, du Tamil Nadu, du Gujarat, de l'Uttar Pradesh ou de l'Haryana.

On peut d'ores et déjà s'interroger sur la durabilité de ces implantations une fois les avantages fiscaux épuisés et si les processus de localisation ne sont pas suivis par des processus de territorialisation (Colletis et Pecqueur, 1995). L'ancrage territorial des firmes dépend à la fois de l'adaptation constante du système productif aux conditions de la concurrence mondialisée et de la singuralisation des avantages compétitifs dans l'économie urbaine nationale. Au-delà des économies de l'agglomération externes aux 
entreprises et internes aux réseaux qui se nouent, les entreprises et les promoteurs immobiliers acquièrent un pouvoir nouveau pour le développement et l'aménagement « durable» des territoires.

\section{B. Multiplication et diversification des forums : le pouvoir croissant des entreprises}

Selon la synthèse effectuée par L. Boussaguet (2004, pp. 227-229), il existe quatre grands types de forums, dont les frontières sont perméables ${ }^{24}$ : (i) le forum scientifique des spécialistes fait évoluer les paradigmes technologiques, l'objectif étant la recherche et l'excellence scientifique; (ii) le forum de la communication est celui de la rhétorique politique ; (iii) le forum des professionnels cherche à faire valoir auprès de sa base et des pouvoirs publics une identité sociale particulière et développe sa propre interprétation de la politique publique sectorielle qui les concerne; (iv) le forum des communautés de politiques publiques est composé d'hommes politiques, de responsables administratifs, d'acteurs sociaux et d'experts reconnus, où se fabriquent les « recettes » politiques. A ces formes d'associations s'ajoutent les réseaux virtuels et les communautés épistémiques fondés sur des liens sociaux, économiques ou scientifiques.

L'ensemble de ces forums, qui s'instituent en nouveaux lieux de pouvoir, ont en commun le partage des usages, des normes, des valeurs et des pratiques entre entrepreneurs, scientifiques, politiques et communicants. Ils révèlent les différentes échelles de l'économie politique relationnelle et de la promotion internationale des territoires spécialisés ainsi que les rivalités de pouvoir entre les acteurs sur un territoire. Devenant des lieux stratégiques de la veille technologique des industries de pointe, ces forums privés semblent avoir pris le relais des pouvoirs publics en révélant de potentiel d'invention et de créativité territoriale et de mise sur pieds de programmes de recherche et développement où fermentent certaines des innovations cruciales pour le développement indien.

Dans la ville de Pune, quatrième pilier des TIC indiennes, les associations intercédant en faveur des TIC renvoient à des configurations différentes. La sociologie et les ressources financières des entreprises membres déterminent l'ampleur et l'échelle des objectifs. Ainsi par exemple, le "Comité aux Technologies de l'Information" de la Mahratta Chamber of Commerce, Industries and Agriculture assiste depuis 1986 les investisseurs dans leurs démarches auprès des pourvoyeurs de réseaux locaux et fait office d'intermédiaire incontournable auprès des autorités urbaines. La Software Exporters Association of Pune est une association professionnelle fondée en 1998 par le directeur général de Persistant qui intervient davantage à l'échelle régionale à propos des arrangements souhaitables des politiques sectorielles afin de maintenir la compétitivité du tissu marathe. Le forum Pune BioIT, fondé en 2004 et rassemblant les communautés scientifiques publique-privée, est quant à lui dirigé par la femme de l'un des plus grands industriels de Pune. Il s'emploie à réunir sous forme de conférence dinatoire les esprits universitaires et commerciaux de la ville afin d'en orienter la bifurcation technopolitaine. La communauté épistémique des informaticiens du Pune Open Coffee Club agit dans le même sens, mais de manière moins évidente. Ces antichambres du pouvoir par leur connaissance de la filière des TIC et leurs réunions régulières (local buzz) prennent progressivement l'ascendant sur les pouvoirs publics en 
orientant certaines décisions stratégiques de l'aménagement urbain. Ainsi, par exemple, sous la pression de plusieurs lobbies dans lesquels figurent les grandes SSII punéites, la priorité est donné aux réseaux de transports collectifs desservant les parcs d'activités informatiques périphériques.

\section{Conclusion} internationaux d'échanges de services informatiques et devenues pour certaines des firmes multinationales (TCS, Wipro, Infosys, Mahindra Satyam), ont directement bénéficié de la coévolution des politiques sectorielles - télécommunication, université, industrie, commerce. Les entreprises ont fleuri essentiellement dans les métropoles et les aires urbaines secondaires et ont renforcé l'aspect numérique de certaines économies régionales - Maharashtra, Tamil Nadu, Andhra Pradesh et Karnataka. Dans le même temps, des forums de diverses natures ont émergés créant ainsi des espaces concrets et virtuels de rencontres entre entrepreneurs et professionnels des services informatiques et solutions logiciels. Progressivement, les échelles centrales et régionales des pouvoirs publics se retirent - par manque de moyens et d'outils adaptés - des aspects directement aménagistes du secteur TIC (réduction des coûts, dérégulation continue de l'économie, création des Zones Économiques Spéciales). Parallèlement, les entreprises ont acquis un rôle croissant, soit directement par la voie de leur Président Directeur Général ou de leur Conseil d'Administration - porte-parole d'un groupement d'intérêts plus vaste, soit indirectement par la présence de ces mêmes personnes au sein des instances de concertation et de décision des aires métropolitaines en devenir.

Ces phénomènes socio-spatiaux mettent en évidence un certain nombre d'enjeux plus largement liés à la mondialisation et à la dynamique du capitalisme contemporain. Les politiques sectorielles indiennes et les rapports de pouvoirs entre l'État - garant d'une certaine équité - et l'espace économique des très grandes firmes informatiques de nationalité indienne semblent en dehors de l'espace immédiatement marchand, sans se soucier d'un territoire néanmoins porteur des potentialités de différenciation futures. En effet, le remplacement des décideurs démocratiquement élus par des décideurs formés au business plans appartenant à des think tanks métropolitains ne risquent-elles pas de faire le jeu des décisions politiques d'attractivité du territoire trop courttermistes, vecteur d'un aménagement à plusieurs vitesses et donc de déséconomies de l'agglomération ${ }^{25}$, contribuant à contrarier la volonté historique des États (centre et régions) et le dessein nouveau des villes indiennes de soutenir territorialement l'innovation? 


\section{BIBLIOGRAPHIE}

Benko G., Lipietz A. (dir.), 1992, Les régions qui gagnent, Districtset réseaux : les nouveaux paradigmes de la géographie économique, Paris, PUF, $424 \mathrm{p}$.

Boussaguet L., Jacquot S., Ravinet P., 2006, Dictionnaire des politiques publiques, Paris, Presses de Sciences-Po, $518 \mathrm{p}$.

Cadène P., 2008, Atlas de l'Inde, Paris, Autrement, 80 p.

Colletis G., Pecqueur B., 1995, « Le rôle des politiques technologiques locales dans la création de ressources spécifiques et d'avantages dynamiques de localisation ", in Rallet A. et Torre A. (eds), Economie industrielle et économie spatiale, Paris, Economica, p. 445-463.

Coris M., Rallet A., 2008 « Les pays émergents à la conquête des marchés mondiaux : une lecture des trajectoires indienne et chinoise dans le secteur du logiciel », Revue de la régulation, $\mathrm{n}^{\circ} 2$, janvier, Varia, http://regulation.revues.org/document2583.html, consulté le 01 juin 2008]

Das G., 2007, Le Réveil de l'Inde, Paris, Buchet-Chastel, 491 p.

Depret M-H., Hamdouch A., 2007, « Changements technologiques, logiques institutionnelles et dynamiques industrielles. Esquisse d'une approche co-évolutionnaire appliquée à l'industrie pharmaceutique et aux biotechnologies ", Innovations, vol. 1,n²5, p. 85-109.

Glaeser Ed. L., 2005, “Reinventing Boston: 1630-2003”, Journal of Economic Geography, vol. 5, n², p. 119-153.

Gumuchian H., Pecqueur B. (dir), 2007, La ressource territoriale, Paris, Economica, 252 p.

Jaffrelot C. (dir.), 2006, L'Inde contemporaine, de 1950 à nos jours, Paris, Fayard/CERI, 963 p.

Kennedy L., 2009, “Large-scale economic and infrastructure projects in India's metropolitan cities: New policies and practices among competing subnational states", The 4th International Conference of the International Forum on Urbanism (IFoU), Amsterdam/Delft, 26-28 November, p. 1055-1066.

Leducq D., 2009, « Secteur TIC et refonte de la gouvernance urbaine: une réflexion sur les nouveaux acteurs de Pune (Maharashtra) », in Didelon C., Ripert B. (eds), « Dimensions sociospatiales des TIC en Inde », Netcom, volume XXIII, n³/4, pp. 221-244.

Lorot P., 1999, Introduction à la géoéconomie, Paris, Economica, 244 p.

Mashelkar R.A., 2008, "Indian science, technology and society: the changing landscape", Technology in Society, 30, p. 299-308.

National Association of Software and Services COMpanies, 2009, Strategic review, New Delhi, 256 p. Planning Commission, 2002, Tenth Five Years Plans (2002-2007): vol. I-II-III, New Delhi,1575 p.

Planning Commission, 1951-1997, 1st to 9th Five Years Plans (1951-2007): chapters "Industry", "Science and Technology", "Education" and "Communication", New Delhi.

Saith A., Vijayabaskar M. (eds), 2005, ICTs and Indian economic development: Economy, Work, Regulation, New Delhi-London, Sage Publications, $474 \mathrm{p}$.

Software Technology Parks of India, 1991-2009, Annual reports, New Delhi, Department of Electronics, Government of India, 667 p. 
Tatemi J., 2005, « Développement du district industriel au Japon : le cas de Kojima », Géographie, Economie, Société, 2005/3, vol. 73, p. 269-288.

Vicente J., 2005, Les espaces de la Net-économie, Paris, Economica, 148 p.

\section{NOTES}

1. Plus de119 millions en 2010 contre 48 millions en 2005.

2. Lui-même indubitablement nécessaire à la reconnaissance du pays comme nouvelle puissance technologique.

3. Les directions générales de ces organismes se trouvent à New Delhi, capitale administrative de l'Union Indienne.

4. Government of India, Planning commission [http://planningcommission.nic.in/index.php, consulté le 19 juillet 2010].

5. Introduction des uns par les autres.

6. Gouvernement central de New Delhi.

7. Indian Institute of Technology (IIT).

8. Concept de l'hindouité né en 1924 avec le théoricien V.D. Savarkar. Il s'agit de l'identité fondée sur les symboles de l'hindouisme, qui pousse à « consommer uniquement indien ».

9. Center for Development in Advanced Computing.

10. Activités numériques, multimédias, télécommunications, logiciel, électro-informatique.

11. En Inde, les sommes importantes, tels que les revenus des entreprises ou les fonds publics dépensés pour les programmes importants, sont exprimées en «Crore(s) ». Un crore de Roupies équivaut à 10 millions de Roupies indienne.

12. Sur un site Internet traduit dans plusieurs langues, dont l'anglais, l'hindi et la langue régionale, les administrés, de la ville à la région, ont accès à des renseignements plus ou moins exhaustifs sur leur localité (master plan, cadastre, emplois), sur des démarches à accomplir en vue de l'obtention de certains documents officiels (acte de naissance, publication des bancs de mariage) ou encore sur le coût de certains services publics, le calcul et le paiement de l'impôt. Internet aura ainsi tendu en une décennie à rendre un peu plus transparente la politique locale.

13. Piloté par la Commission au Plan en charge du développement économique, le « règne des licences » a été en vigueur en Inde de 1950 à 1991. Pour augmenter ou diversifier sa production, chaque entreprise devait solliciter l'autorisation de l'administration, ce qui était source de lenteurs bureaucratiques et d'une nouvelle forme de corruption.

14. Les entretiens semi-directifs ont eu lieu au cours de trois séjours de recherche, en 2007, 2008 et 2009 .

15. Emprunt à George Benko et Alain Lipietz, 1992.

16. Calcutta a été renommée Kolkata en 2001.

17. Conçu en 1896 par Jamsetji Nusserwanji Tata et fondé en 1909, l'IISc recrute l'élite estudiantine pour des formations de $2^{\text {nd }}$ et de $3^{\text {ème }}$ cycles en sciences, allant de la biochimie à l'aérospatial en passant par l'électronique et les sciences physiques. Le but est surtout à travers des doctorats et post-doctorats d'excellence de former des chercheurs qui sauront se distinguer sur la scène académique mondiale.

18. Association d'anciens étudiants des grandes écoles ou des universités prestigieuses sur le modèle de la Old Boys Society anglo-saxone.

19. Explorant la niche que représente la filière audiovisuelle-multimédia, cette évolution de la politique régionale du Maharashtra dédiée aux TIC est une émanation directe d'un sous-comité de la Maratha Chamber of Commerce (MCCIA) de Pune. Elle est révélatrice d'une volonté de lier la technologie aux contenus et de soutenir les formations et les créations d'entreprises dans les 
domaines de la création d'illustrations numériques et graphiques en 2D et en 3D, des effets spéciaux et de l'édition de films.

20. Soutien financier offert par l'État central à travers le STPI ; zones créées par les Agences publiques d'aménagement et de Développement Régional.

21. BPO.

22. Special Economic Zones.

23. Il s'agit d'une carte inédite réalisée à partir de la publication officielle des approbations trimestrielles du Commissariat au Développement des ZES, placé sous l'égide du Ministère indien de l'Economie.

24. Reprise d'une partie des travaux d'Eve Fouilleux.

25. L'agglomération des SSII donne lieu à une croissance urbaine dépassant le bassin d'activité et d'emploi. En l'absence d'anticipation des municipalités via des plans de développement et de déplacements urbains, cette croissance - plus ou moins soudaine - entraîne des congestions et des pollutions sonores, olfactives et visuelles. Ces phénomènes provoquent à la fois des déséconomies d'échelle internes à l'entreprise et des déséconomies de l'agglomération externes à l'entreprise mais communes au territoire.

\section{RÉSUMÉS}

La filière indienne des TIC offre, du point de vue des relations géoéconomiques, un terrain d'étude particulièrement intéressant pour l'observation du retournement progressif des jeux de pouvoir entre les firmes et les États. Le climat favorable aux affaires a été grandement favorisé et consolidé par des acteurs publics indiens multiscalaires. Toutefois, la part grandissante et indirecte que prennent les entreprises informatiques dans l'aménagement des territoires urbains est significative d'une inversion de plus en plus visible des prérogatives et des rôles entre acteurs publics et privés.

Indian ICT sector is an interesting field of study to understand the evolution of the geoeconomical relationships between Firms, State(s) and territories. The favorable business climate has been implemented and consolidated by the multilayered public actors, from national to urban scale, through regional one. Nonetheless, one can mention that Information Technology enterprises are taking a growing and indirect involvement in urban and regional planning, which is significant of the continuous reversal of powers between public and private actors.

Hinsichtlich geoökonomischer Beziehungen ist der Sektor der Informations- und Kommunikationstechnologien in Indien ein besonders interessantes Untersuchungsgelände für die Beobachtung der allmählichen Trendwende der Machtspiele zwischen den Firmen und den Staaten. Das Milieu, das die Geschäfte begünstigt, wurde von indischen öffentlichen Akteuren in unterschiedlichen Skalen unterstützt und gefestigt. Jedoch, ist der wachsende und indirekte Anteil der Informationsunternehmen in der Raumordnung der indischen städtischen Territorien kennzeichnend von der immer sichtbaren Umkehrung der Vorrechte und der Rollen zwischen öffentlichen und privaten Akteure. 
INDEX

Mots-clés : État, firmes, Inde, Technologies de l'Information et de la Communication, territoires Schlüsselwörter : Firmen, Indien, Informations und Kommunikationstechnologien, Staat, Territorium

Keywords : firms, India, Information and Communication Technologies, State, territories

\section{AUTEUR}

DIVYA LEDUCQ

ATER - Doctorante - Université Lille Nord de France - Laboratoire TVES (EA 4477) - Lille 1 - Cité scientifique - UFR de Géographie et Aménagement - 59655 Villeneuve d'Ascq Cedex -

divya.leducq@gmail.com 